\title{
Aspects of Voice Irregularity Measurement in Connected Speech
}

\author{
Adrian Fourcin \\ University College London, London, UK
}

\section{Key Words}

Voice irregularity measurement $\cdot$ Connected speech

\begin{abstract}
Applications of the use of connected speech material for the objective assessment of two primary physical aspects of voice quality are described and discussed. Simple auditory perceptual criteria are employed to guide the choice of analysis parameters for the physical correlate of pitch, and their utility is investigated by the measurement of the characteristics of particular examples of the normal-speaking voice. This approach is extended to the measurement of vocal fold contact phase control in connected speech and both techniques are applied to pathological voice data.
\end{abstract}

Copyright $\odot 2009$ S. Karger AG, Basel

\section{Introduction}

Although we use voice as a dynamic time-varying source of vocal tract excitation for all speech communication, connected speech is not currently the preferred signal for objective measurement in the voice clinic. In principle, however, it ought to provide crucially important data in the definition of what makes speech normal and serve as a central reference in the understanding and management of abnormal spoken communication. In particular, the rapid successions of different articulatory controls that are essential for the production of efficient speech sound sequences subject laryngeal phonatory mechanisms to transient inputs that continually test their function to a depth that is quite unattainable by the sole use of sustained vowels.

The present brief discussion is concerned with the quantitative clinical use of vocal fold frequency measurement in connected speech. The essential aim is to explain the basis of a simple approach that, in the first instance, makes use of auditory criteria in the definition of physical differences between normal regularity and unacceptable irregularity in voice pitch. Figure 1 shows aspects of the voiced component of a single word spoken with a falling intonation that, initially, has a smoothly changing vocal fold closure repetition frequency. Fx in the figure refers to the 'instantaneous' frequency of vocal fold vibration (in contradistinction to F0 that is ordinarily obtained from temporal windowing). The final part of the utterance, however, has obvious markedly irregular variations in cycle-to-cycle frequency ('pitch'), peak amplitudes and also contact phase durations. All of these factors, together with others that are not so readily visible, may contribute in their different ways and different degrees to the percept of roughness that ordinarily pervades our auditory appreciation of day-to-day speech. Only pitch variability, however, has begun to be adequately linked to psychophysical and psychophonetic measurements.

\section{KARGER}

Fax +4161306 1234

E-Mail karger@karger.ch

www.karger.com
(C) 2009 S. Karger AG, Basel

$1021-7762 / 09 / 0613-0126 \$ 26.00 / 0$

Accessible online at: www.karger.com/fpl
Prof. Adrian Fourcin

68 Tavistock Court

Tavistock Square

London WC1H 9HG (UK)

Tel. +44 7770364 636, Fax +44 207383 2039, E-Mail a.fourcin@ucl.ac.uk 
Fig. 1. Micro-intonational voice regularity and irregularity. Sp = Speech pressure waveform - compression positive; $\mathrm{Lx}=$ EGG waveform with ELG polarity - contact positive $[1,2]$; Tx = vocal fold cycle duration between successive $\mathrm{Lx}$ contact markers; $\mathrm{Ax}=\mathrm{Sp}$-positive pressure peak after Lx contact marker; Fx = 'instantaneous frequency' - derived from Tx; $\mathrm{AxFx}=$ composite 'intonation' trace made by width modulating the cycle-by-cycle vocal fold instantaneous frequency trace with the corresponding Sp peak values; $\mathrm{Qx}=$ percent ratio to Tx of Lx contact duration $-70 \%$ below $\mathrm{Lx}$ waveform peak. The sampling rate used to determine Tx and Fx is $1 \mathrm{MHz}$.

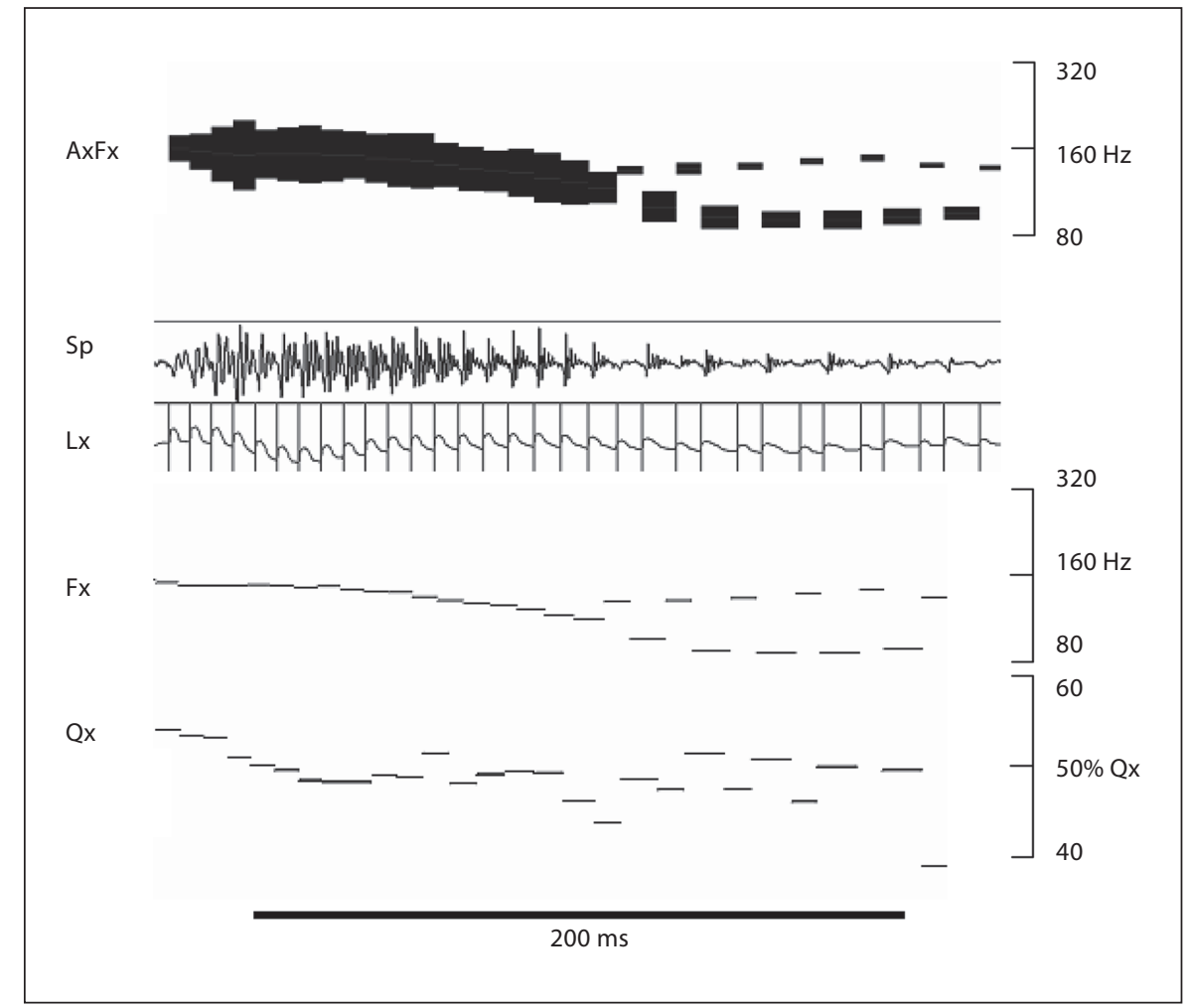

Table 1. Just detectable audible differences, DLs, in frequency for normal listeners responding to pure tones, sustained vowels and connected speech

\begin{tabular}{lllll}
\hline Sound type & Pure tone & Vowel & Connected speech \\
\cline { 2 - 3 } & & sustained & fall or rise & \\
\hline Frequency range, Hz & $200-1,000$ & $200-600$ & 100 & $60-180$ \\
DL semitones & $0.07-0.12$ & $0.02-0.05$ & 1.3 & $1-3.3$ \\
\hline Source & Nelson et al. [4] & Wier et al. [6] & Klatt [7] & 't Hart [8] \\
& Vance [5] & Moore et al. [3] & & \\
\hline
\end{tabular}

\section{Voice Pitch}

Table 1 gives a summary of the pitch perceptual sensitivities that are related to two broad classes of sounds sustained and varying. The analysis of the physical attributes of steady vowels provides a mainstay for current laryngological voice assessment, and it is important to note in the present context that the normal speaker's control of sustained voice pitch precisely matches the normal listener's auditory ability to discriminate voice pitch changes [3-8]. For sustained vowel assessment, this link between production and perception in respect of this dominant aspect of voice quality is an intrinsic supportive aspect of present clinical practice.

The values of difference limen (DL $\equiv$ just noticeable difference) for the perception of voice pitch changes in connected speech are so markedly different from those found for sustained vowel perception, however, that a radically different auditory perceptual processing mechanism must be supposed to exist in our ordinary day-today use of spoken voice - both as listeners and also as speakers. The greater auditory integration times needed 


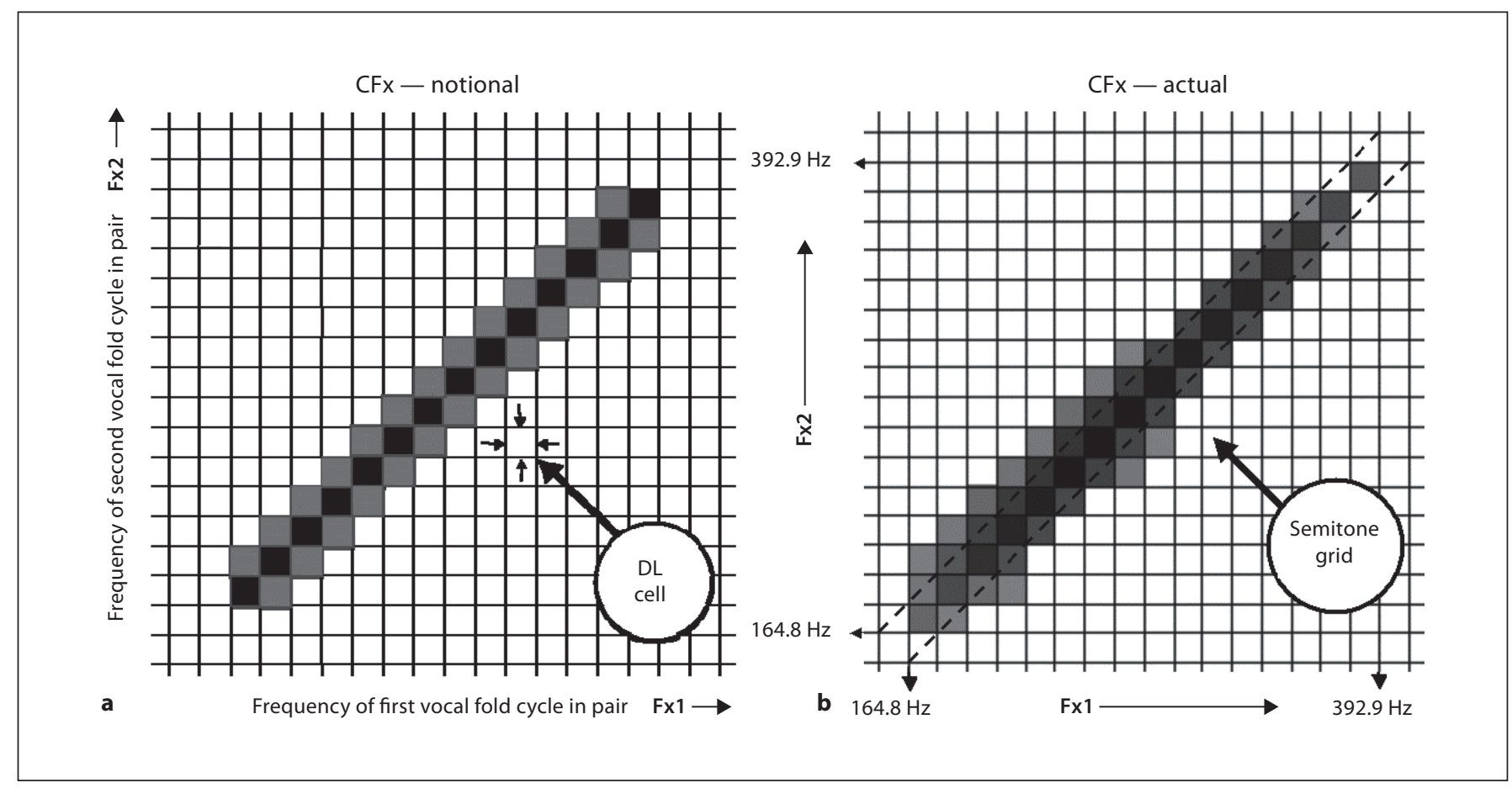

Fig. 2. a A perceptual grid with superimposed notional production. The spacings between adjacent vertical lines and those between adjacent horizontal lines are intended to correspond to a just auditorily distinguishable difference in voice frequency (DL). The black filled bins show the core of the result if a voice sample with no discernable cycle-to-cycle variability were to be analysed in these terms on a cycle pair basis. The light gray bins relate to the occasions in this smooth Fx contour for which there are 2 DL

for DLs in singing, for example, would not enable the rapid processing required for normal speech comprehension. Although the DLs for sustained vowel pitch perception are not explicitly taken into account in voice analysis algorithms [9], they are attractive in providing a possible guide to the definition of protocols for the quantitative analysis of voice in connected speech. Spoken voice variability typically occurs often on a cycle-to-cycle basis within a speech sound and there are no extant perceptual measurements that provide a means of estimating their perceptual importance. The normally hearing speaker, however, continually monitors the nature of his/ her speech and uses auditory feedback to signal the need for correction. It appears that it may be profitable to make use of an aspect of this process in physical measurement. In the limit, the auditory processing of the client and clinician is the final arbiter of unacceptable variability. changes in pitch within a successive pair of cycles. b An actual production on a perceptual grid. Expanded CFx crossplot analysis of a 2-min sample of normal speech, produced by an occupational voice user, with a superimposed perceptual semitone grid and dashed reference double semitone DL lines. 15,250 vocal fold cycles have been analysed and 488 have fallen outside the cells marked by the dashed upper and lower DL lines, giving an index of variability Ix of $3.2 \%$.

Before this can be done, however, there is a basic difficulty arising from the intrinsic nature of voice irregularity that must be clarified. The classic measurements made of perceptible pitch changes that are shown in table 1 are all based on the perceptual discrimination of whole pitch contours. The problem to be solved when dealing with the analysis of vocal frequency changes in ordinary speech arises from the need to provide an objective frame that enables changes from moment to moment in a given single utterance to be quantified.

\section{Variability Measurement}

If the timed closure markers of figure 1 are used to produce a cycle-by-cycle string of estimates of successive vocal fold vibrational frequencies $(\mathrm{Fx})$ then these can be used to show and to measure cycle-to-cycle variability in 
a way that is relatable to pitch. In figure 2 this has been done in a purely illustrative way using a grid of spaced lines that are separated by distances that are notionally related to DL pitch differences that apply to connected speech. This construction of fixed DL cells does not allow for the variation in DL values that may occur as a function of frequency and it is intended only to apply to the mode of voice frequency control that is associated with conversational speech. The singing voice, for example, would require more closely spaced DL bin lines in its analysis in order to cater for the greater precision of pitch control in production and in perceptual monitoring.

The variability that is potentially open to measurement is one that only extends over adjacent cycle pairs and, within each cycle of each pair, it is only necessary for the 'instantaneous' frequency, Fx, of that cycle to fall within a bin for it to be included. Figure 2 has a notionally well-controlled sample of voice pitch data superimposed on its grid lines - with variability in cycle-to-cycle variability that never exceeds 2 DLs. The central diagonal core of this crossplot (CFx) contains only those cycle pairs whose members differ in Fx by less than 1 DL. The real test of this approach, in general and at this level of detail, however, can only properly come from its use with real speech data.

Figure $2 \mathrm{~b}$ has the same basic construction as that of figure 2a, but now the grid lines are spaced by half-tone $(6 \% \mathrm{Fx})$ intervals and the superimposed data have been derived from a recording of a read text by a speaker who is used to taking care of her voice. This association of an actual voice recording analysis with a perceptual grid gives a correspondence between production and perception that is not likely to be a matter of coincidence. It enables a simple metric of irregularity to be used that can be employed for the study of pitch control and vocal fold closure timing irregularity (see Fx in fig. 1) in the connected speech of both normal and pathological voices. When the separation between successive vocal fold instantaneous frequencies ( $\mathrm{Fx}$ ) exceeds 2 semitones then the corresponding pair of values falls into a cell that is outside the main central diagonal of this figure and the total of all of these occurrences can be used as a simple measure of irregularity, termed IFx and expressed as a percentage of the total number of cycles in the whole sample. IFx is $3.2 \%$ for this 2 -min sample of a read text, so $96.8 \%$ of the vocal fold cycles in the whole recording are associated with a cycle-to-cycle variability less than or equal to 2 semitones. Although the rationale basic to this measurement method has evolved interactively with laryngologists, speech and language therapists, speech sci-

Voice Irregularity Measurement in

Connected Speech entists and phoneticians over a number of decades and the implementation that has just been described has been in clinical use for more than 2 decades, its rationale has not previously been spelled out. It has recently been the object of an excellent normative study by Prof. Ptok et al. of Hannover [11]. The approach is of use in the study both of aspects of normal speech production and also of the pathological voice.

Figure $3 \mathrm{c}$ shows the average CFx crossplot for a particular group of 14 English speech and language therapy students. Their work has not required that they acquire exceptional speaking skills, and, paradoxically they are not given any special voice productive training. They are concerned with effective speech communication and are not called on to use expressive voice. Their mean vocal fold frequencies are similar and so also are their closure irregularities; the mean irregulatory score (IFx) of $6 \%$ is low and indicative of low closure timing irregularity.

Figure $3 \mathrm{a}$ is based on the analysis of the texts read by a quite different but similar age group of women dramatic arts students. A major objective of their training is concerned with voice production and the acquisition and control of expressive voice. It is immediately obvious from figure 3 a that this group has a greater voice frequency range than the group in figure $3 \mathrm{c}$ and it is also the case that their average IFx of $11 \%$ is greater than that for the speech and language therapist group. One important aspect of voice control is related to voice quality, as well as voice frequency range, and an ingredient of contrastive phonation types is closely linked in English to the use of creaky voice. The approx. 2-min read passage used by these students was designed so that there was no opportunity given for the use of 'character' voices but, even so their training, after 2 years, has set them apart from the therapy students. The male dramatic arts students have an IFx score of approx. $11 \%$ in figure $3 \mathrm{~b}$, and this also at first sight is surprising since it is higher than may be found for some other young men. These measurements are part of an ongoing study and although it is premature to draw firm conclusions, it is possible that the daily emphasis given to voice control has made these speakers, as for their women colleagues, use more creak than their contemporaries. It does not follow that either of these student groups has developed voice pathology as a result of their training [10] since the structure of their CFx plots is not abnormal.

A rough rule of thumb has emerged from the clinical use of the CFx plot measurements that gives as a first guideline that an IFx of $10 \%$ is at an approximate borderline between good regular phonation (see also the quan- 


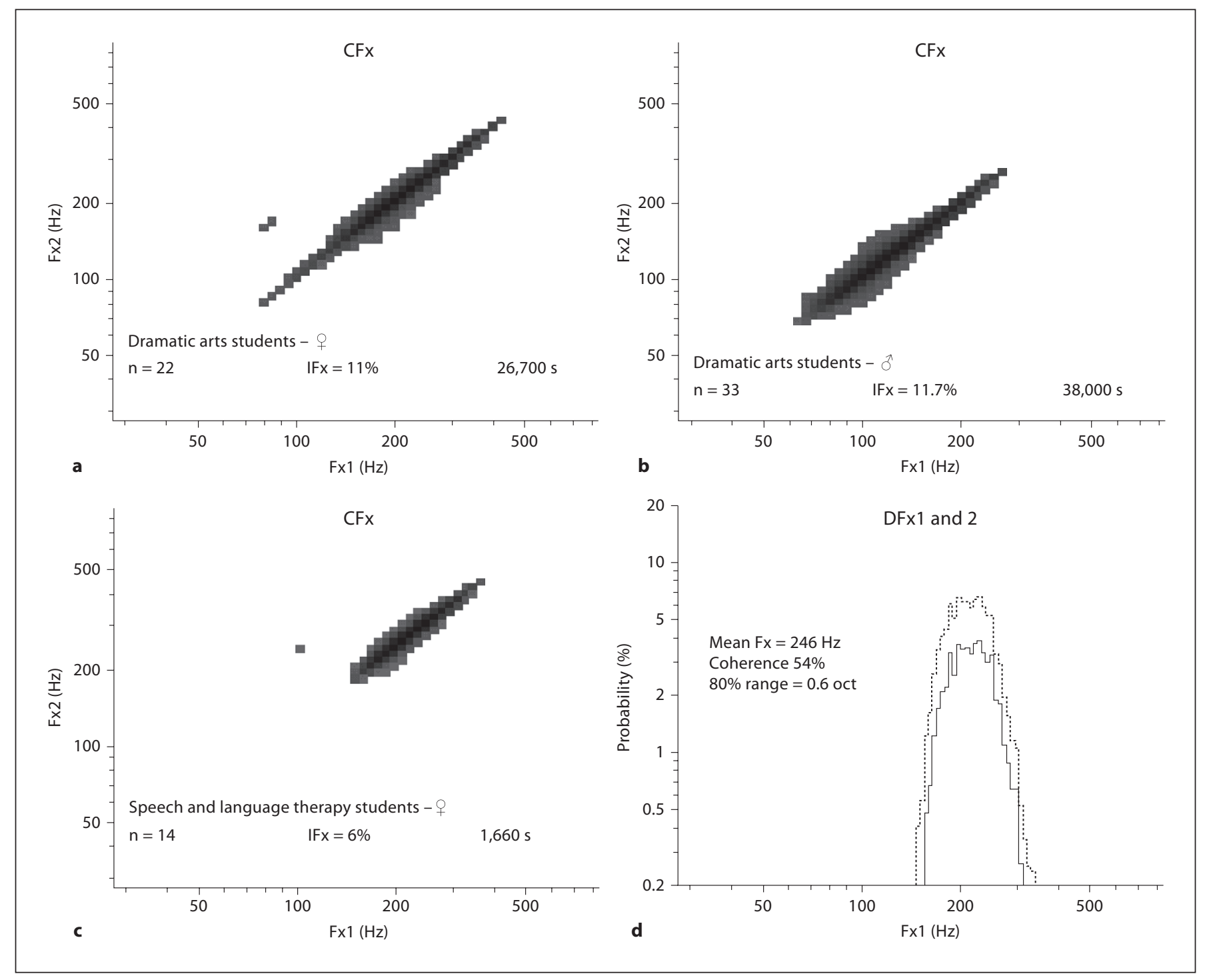

Fig. 3. CFx examples for different normal speaker groups. a-c The speech and laryngograph data samples for each member of the three separate groups of speakers have been grouped together and analysed as single files. This technique gives an overview of the average irregularity that is characteristic of the speaker class. d Based on the same data as c, the probability distribution of Fx occurrences is shown using 3\% quarter tone wide bins. DFx 1 gives only the probabilities of occurrence for every vocal fold cycle in

titative normative work by Prof. Ptok et al. [11]). Voice pathologies are likely to be linked to much higher values of IFx and irregular CFx plots.

Figure $3 \mathrm{~d}$ shows two data representations that are related to figure $3 \mathrm{c}$. The dotted outside plot, DFx1, makes use of all the Fx samples in the voice sample to show the range and way that their frequencies are distributed

the whole sample whilst DFx2 gives the probabilities for pairs of successive cycles falling within the same $3 \%$ Fx bin. DFx 2 is linked to the centre diagonal of the corresponding CFx analysis of the data, but like DFx1 makes use of a smaller bin. Both CFx and the DFx plots start at $27.5 \mathrm{~Hz}(\mathrm{~A} 0)$ and end at $880 \mathrm{~Hz}$ (A5). Time given in seconds refers to the duration of the whole amalgamated sample.

whilst the inner DFx2 plot only shows the distribution of successive Fx samples that occur in pairs falling within a single DL analysis bin. DFx2 is related to the inner diagonal bins of the CFx plot and the coherence value is the ratio of these 'regular' cycles to the size of the whole sample. 


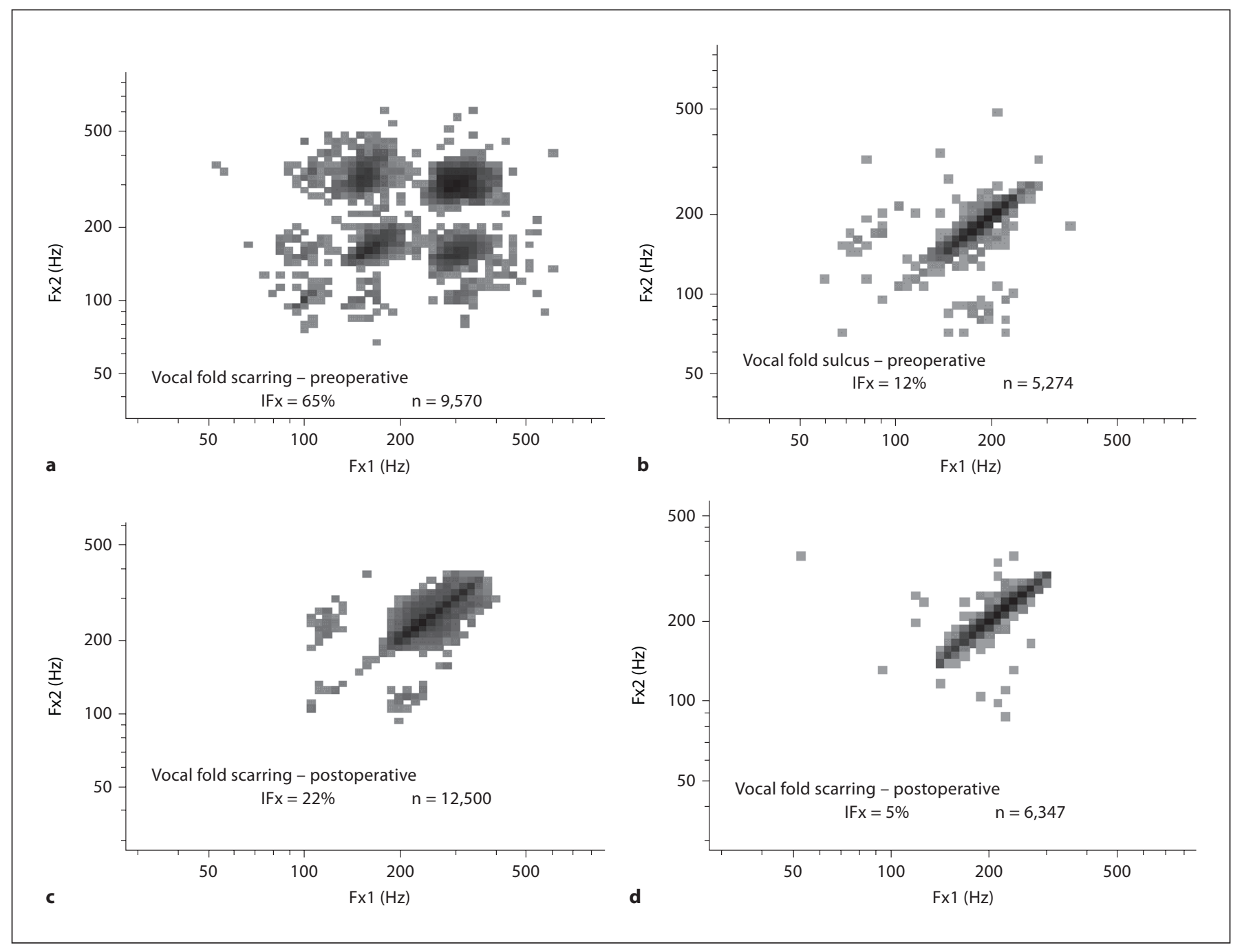

Fig. 4. Pathological voice examples of CFx analysis. a, c The first pair of examples on the left concern a single patient whose primary voice quality disability arises from vocal fold closure timing (Fx) irregularity. In these cases the use of the IFx index is of direct management utility and relates to the auditory impression of the associated voice quality. There may, however, be additional fac-

tors that influence voice quality. $\mathbf{b}$ is linked to a poorer voice quality than might be supposed from an IFx of $12 \%$ and $\mathbf{d}$ is associated with a change to almost normal voice quality. (Data for $\mathbf{a}$ and c by courtesy of Mr. McGlashan FRCS, Nottingham, and data for b and $\mathbf{d}$ from Dr. Coulombeau and Dr. Pérouse, Lyon).

Figure 4 shows two sets of CFx crossplots for the comparison between the preoperative and postoperative vocal fold closure timing regularity analyses for 2 phonosurgical patients. Figure $4 \mathrm{a}$ has four main lobes, two on the main diagonal at $170 \mathrm{~Hz}$ and the main concentration at $300 \mathrm{~Hz}$. It is possible immediately to infer that, for a 35 -year-old woman, the voice will present as being unnaturally childish. The presence of the side lobes at $[150$, $300 \mathrm{~Hz}]$ and $[300,150 \mathrm{~Hz}]$ on either side of the main diagonal indicates a high probability of diplophonic octave

saccades that will give a strong sensation of roughness. The second lobe in the main diagonal, at $170 \mathrm{~Hz}$, is within a more normal pitch range for the speaker but once more is linked to symmetrical side lobes at about [100, $170 \mathrm{~Hz}$, and these will also give rise to perceived although lesser irregularity. Postoperatively, the vibrational CFx pattern is far less complex with very little irregular diplophonia at $[205,120 \mathrm{~Hz}]$ and $[115,245 \mathrm{~Hz}]$ and a main lobe now centred on $230 \mathrm{~Hz}$. This main lobe, however, is quite broad and extends for \pm 7 semitones and this indi- 


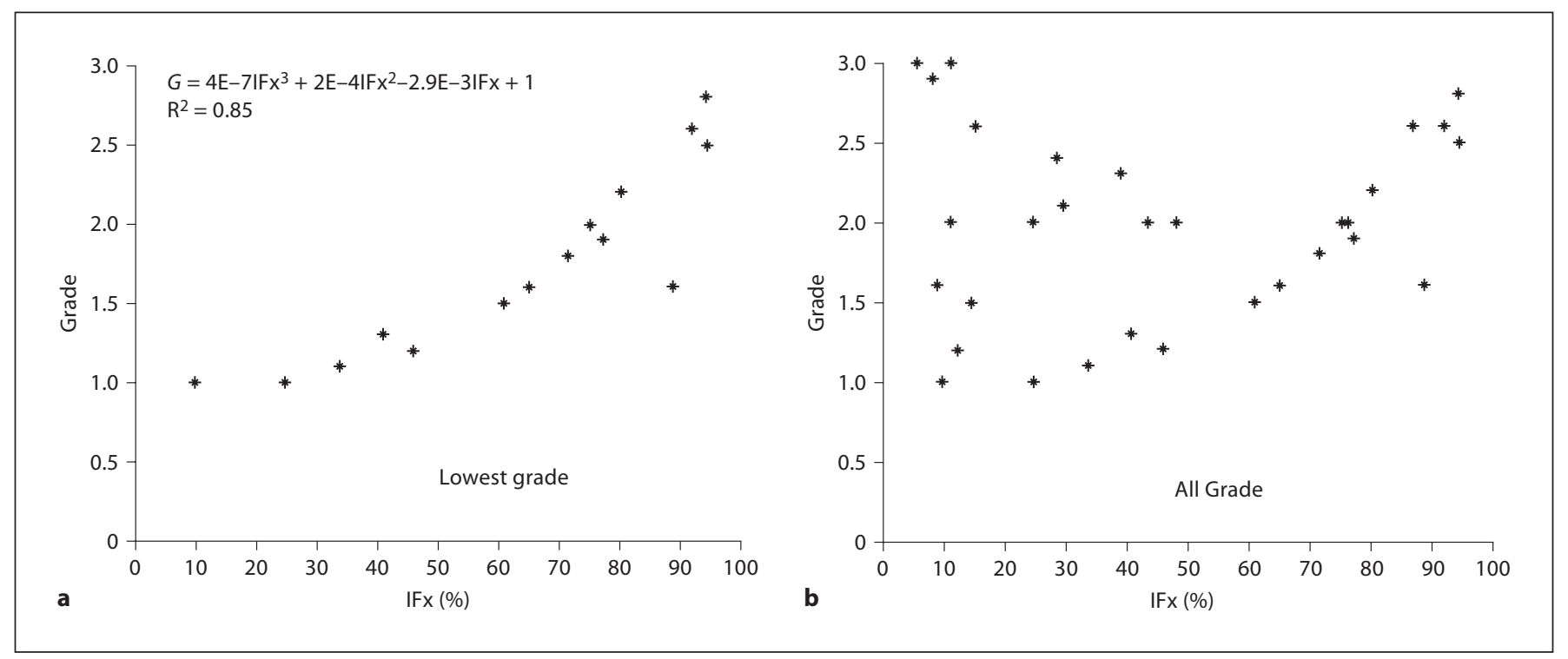

Fig. 5. Grade from GIRBAS evaluations and CFx measurements. This comparison comes from grade evaluations made by Dr. Fresnel as part of a study organized by Prof. Dejonckere et al. [13]. Assessments for 32 patients are shown. One half of the IFx values from CFx analyses lie on the lower border of the set of grade values (a). For these values of grade the dysfunction seems likely to be

cates that, although there has been a considerable improvement with a change in IFx from 65 to $22 \%$ in closure timing regularity, the voice is still perceptually very rough.

The data for the second set of figure $4 \mathrm{~b}$ and $\mathrm{d}$ relate to a quite different degree of dysphonia. There is a little change in the main modal frequency from 190 to $210 \mathrm{~Hz}$ and initial IFx of $12 \%$ is already near to normal.

The postoperative CFx plot shows a real improvement in regularity, but in the auditory comparison of the samples there appears to be at least one other perceptual factor that should be taken into account and this is considered in the discussion of figure 7.

The GRBAS scale was originally introduced for the perceptual grading of sustained vowel data [12], but it has been widely established for use in the voice clinic with connected speech. A main objective of a study organized by Prof. Dejonckere et al. [13] was to calibrate an extended version of the scale, GIRBAS, and compare its findings with objective analysis by the cooperative work of pairs of clinicians in 5 institutes in 3 European countries. Two types of spoken material were used, for the objective analyses, the sustained vowel as in the English word 'are' $[\Psi:]$ and a short sentence (continually voiced and with neither fricatives nor voiceless plosives). purely related to closure irregularity. The inference from these results is that for the other half of the voice recordings (b), there are other sources of dysfunction that contribute to the grade assessment. Walzak et al. [10] is the source for the GIRBAS study; Prok et al. [11] discusses other sources of irregularity and their measurement.

The subjective GIRBAS appraisal was based on a much longer sample of running speech for each patient and recorded from the consultation. In one centre, in Paris, Dr. Fresnel simultaneously made CFx analyses during the recording of the consultation conversation. The results of the IFx values from these analyses are shown in figure $5 \mathrm{a}$ and $\mathrm{b}$, plotted together with the $G$ (grade) ratings for each patient.

In figure $5 \mathrm{a}$, the lowest value of $G$ (the most reliable of the GIRBAS grades) has been plotted against the associated value of IFx. This provides a way of selecting $17 \mathrm{pa}-$ tients for whom it is highly probable that Dr. Fresnel made the grading on the basis of purely temporal vocal fold vibrational irregularity. There is, in this graph, a quite well-defined trend to assign higher $G$ values to recordings with greater IFx percentages.

Figure $5 \mathrm{~b}$ has been plotted using the $G$ and IFx percent data to include the further 14 patients previously eliminated. To differing degrees these additional patients are not adequately represented, in the present objective terms, by the value of their associated IFx percent measurements. In some cases, figure $5 \mathrm{~b}$ shows that it is possible for a patient's voice to have a very low value of IFx but to be assigned a high $G$ value. Subsequent to this earlier work, voice clinicians (Mr. McGlashan in Nottingham 


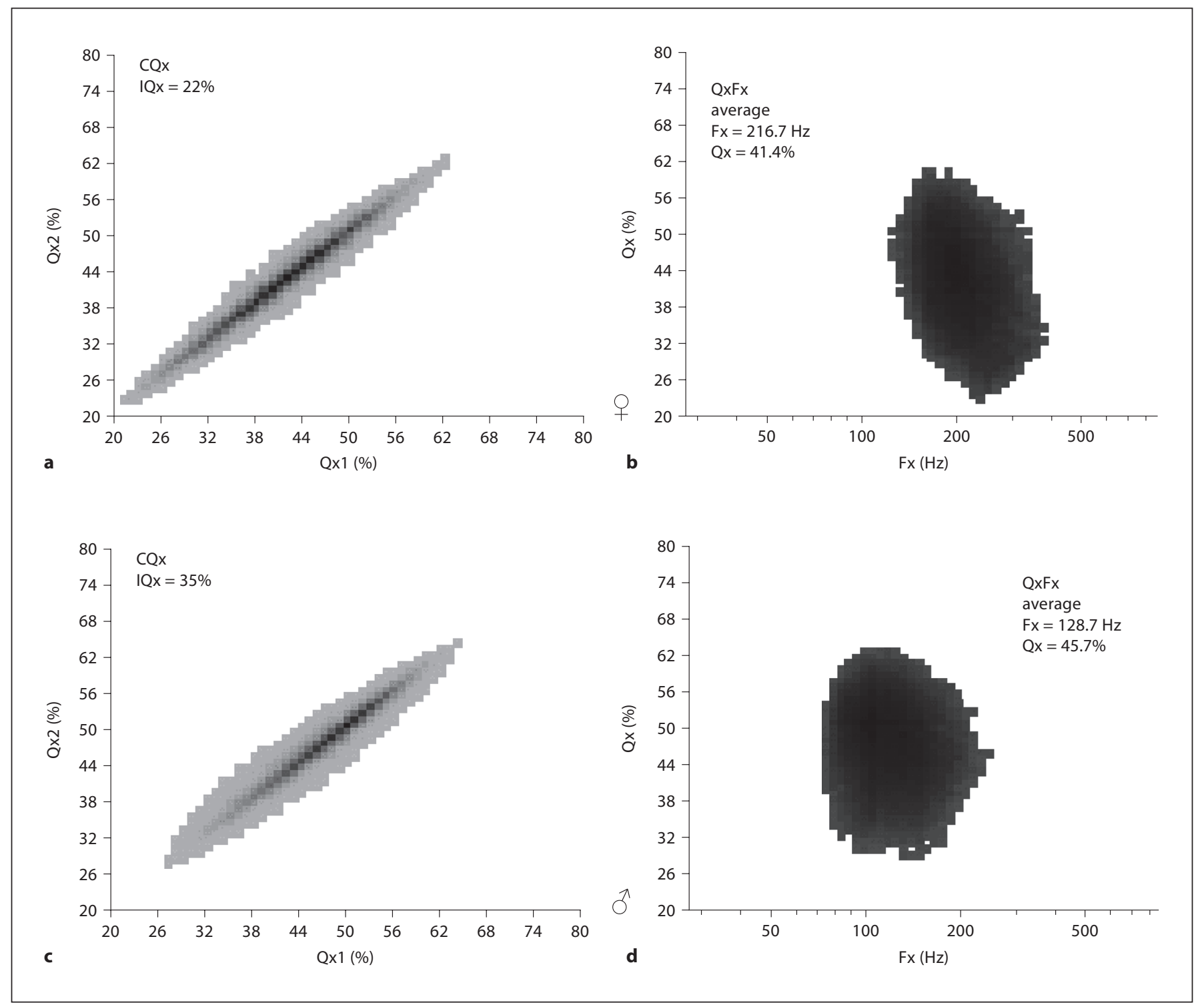

Fig. 6. CQx and FxQx examples for two normal speaker groups. The data samples for each member of the separate groups of speakers have been grouped together and the resulting amalgamation has been analysed as a single file. a, c The paired cycle-to-cycle variabilities arising from changes in vocal fold contact phase duration. This aspect of voice production has proved to be of clinical importance and these samples give an indication of variation that might be found with normal larynges. $\mathbf{b}, \mathbf{d}$ It is shown how these men and women speakers, on average, control their contact phase, Qx, as a function of vocal fold frequency, Fx. There is a distinct gender contrast. This is likely to prove to be language independent since it arises primarily from anatomical differences. The CQx and $Q x$ plots start at $Q x=20 \%$ and end at $Q x=80 \%$ with 60 equally spaced $1 \%$ bins, whilst Fx, as before, is in the range A0-A5. and Prof. Ptok in Hannover) have observed that considerable voice quality improvement can result from treatment (phonosurgery and electrostimulation, respectively) even though IFx percent values before and after treatment were essentially the same. A major difference between the objectively measured voice qualities before and after treatment for these patients appeared in the contact phase analyses of the Qx measurement shown in figure 1 [2] rather than in vocal fold vibratory closure epoch timing control. The tentative conclusions to be drawn in regard to the data presentations of figure 5 are that a little under half of Dr. Fresnel's group had voice quality 


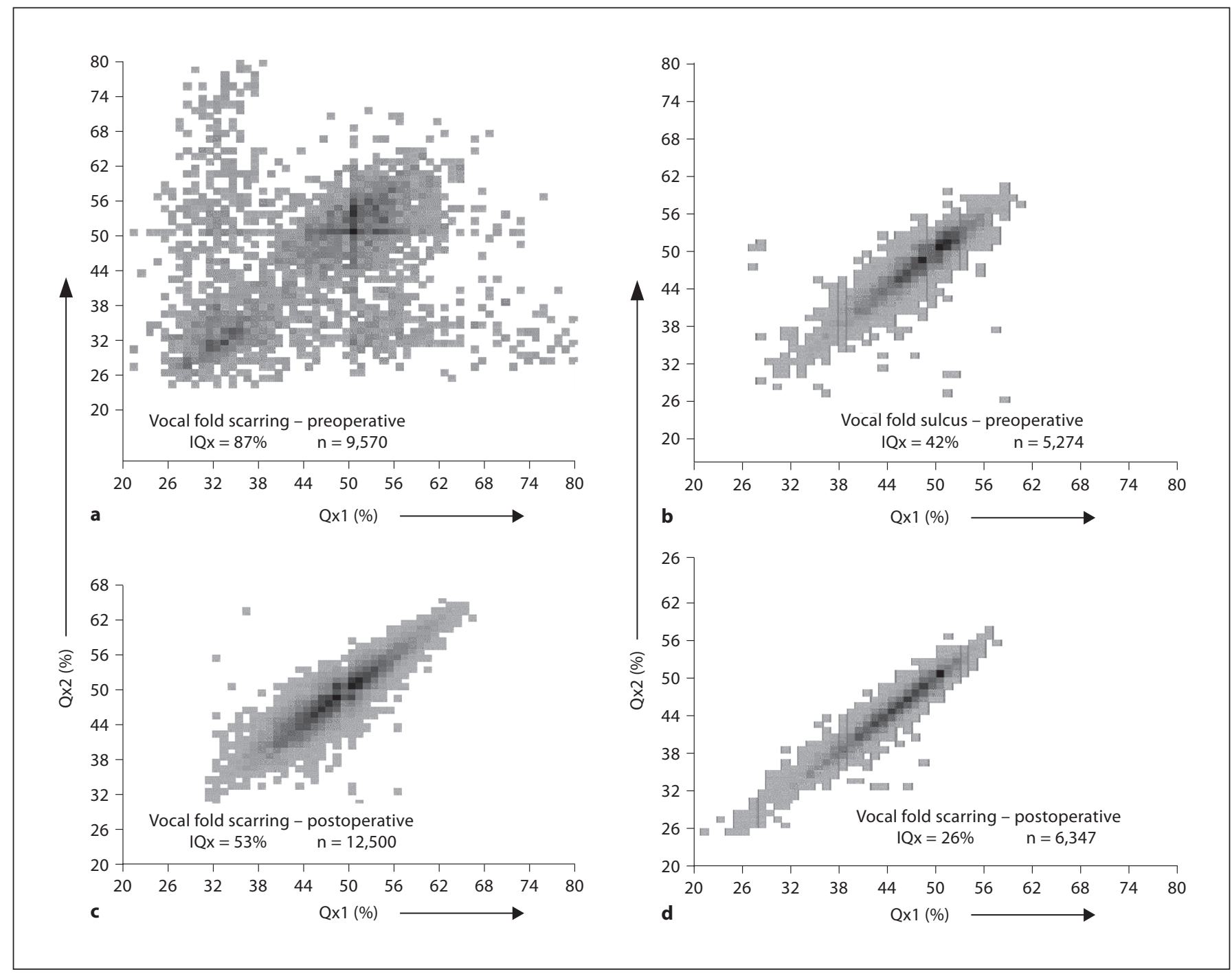

Fig. 7. Pathological voice examples of CQx analysis. Figure 5 indicates that, for that data set, it was likely that half of the voice cases had purely temporal, Fx, vocal fold closing variabilities; for the other cases, additional factors were possibly involved. These

characteristics that had both closure timing as well as other factors, particularly perhaps contact phase, contributing to their dysphonias. If contact phase is an important descriptor of voice quality then it must be a feature that is perceived by and productively controlled by normal speakers. In the context of the present work, this can be examined by making use of Qx crossplot analyses.

Figure $6 \mathrm{a}$ and $\mathrm{c}$ shows the contact phase crossplots, $\mathrm{CQx}$, for the two groups of dramatic arts students for whom the CFx analyses were plotted in figure 3. CQx is

two extensions of the analyses of figure 4 give examples of the improvements in contact phase $(\mathrm{Qx})$ irregularity that may result from phonosurgical intervention, and that occur in addition to the improvements that are linked to closure epoch timing.

constructed in the same way as CFx with the important difference that the analysis bins are entirely heuristic and depend for their validity on the empirical evidence that normal speakers control their use of expressive vocal fold contact phase in ordinary speech in a consistent fashion. The two values of IQx shown in the figures are derived, in the same way as for IFx, with reference to a central core of bins, in CQx. The 'DL' boundaries in CQx are also set by the definition of the bin size that is used. For these figures the bin size is $1 \%$ and the boundary beyond which irregularity is considered to exist is, as before, at \pm 2 bins 
or $2 \%$ on either side of the core diagonal. For the women students in figure $6 \mathrm{a}$, the implication is that nearly $80 \%$ of their vocal fold vibrations are within an IQx percent variability of approx. $20 \%$; the speech and language therapy students, however, have an average IQx of approx. $30 \%$. For the men, the corresponding coverage is about $65 \%$ for an IQx percent variability of $35 \%$.

Figure $6 \mathrm{~b}$ and d illustrates an important gender difference in the control of Qx between young men and young women. Men tend to have the same average contact phase control across their normal voice speaking frequency range. Many (although not all) young women tend to the production of a smaller contact phase ratio in the upper part of their voice register [14]; this is shown here by the downward gradient of the women's average QxFx distribution. It is worth noting, however, that all these dramatic arts students control their overall use of voice timing in connected speech in the same way, so that voice takes up almost exactly $50 \%$ of the total speaking time. The other half of the total speaking time is taken up by voiceless fricatives and silences (an effect that has also been observed for other languages than English [2]).

Applications of this CQx analysis to the pathological voice data of figure 4 are shown in figure 7 . A comparison of figure $4 \mathrm{a}$ and $\mathrm{c}$ with figure $7 \mathrm{a}$ and $\mathrm{c}$ shows that there is an obvious possibility that both closure timing and contact phase timing regularity changes have contributed to this patient's voice improvement. The phonosurgical intervention has reduced IQx from 87 to $53 \%$, but is still greater than the $20-30 \%$ for the women students. Figure $7 \mathrm{~b}$ and $\mathrm{d}$ also shows an improvement in Qx control following phonosurgery, with a final value of IQx that is closer to what would normally be expected (and smaller than the IQx of fig. 6c). Mr. McGlashan has applied the Voice Handicap Index assessment technique to the assessment of patient voices and finds a striking correlation with changes in IQx, and these brief particular examples also indicate that contact phase control is exerted by the speaker and may, in consequence, be linked to voice quality improvement perceived by the listener.

\section{Discussion}

Connected speech not only has obvious relevance to voice use but is also capable of being used as the basis for objective voice quality analysis. At present EGG/Lx electrolaryngograph techniques provide an effective basis for signal processing, but the ear's auditory analysis will eventually be sufficiently well modelled so that acoustic data alone may be sufficient to enable the physical application of perceptual processing.

A special emphasis has been given here to the use of auditory psychophonetic measurements in defining analysis criteria and this is likely to be merely the beginning of further developments that will make more use of accumulating neurophysiological observations that relate to the mechanisms of temporal pitch processing [15]. The remarkable human auditory ability to adjust its effective integration time to suit the analysis of widely different acoustic inputs, so that sung voice pitch may be heard with tenfold greater precision than that for connected speech (table 1), will have to be incorporated explicitly in the voice analysis algorithms of the future.

It was not evident that contact phase variation might be such an important component of connected speech voice quality until clinical observation resulted in data that could only be given this explanation, and the fact that speech is controlled in this regard also implies that interactive voice therapy procedures may benefit from appropriate related biofeedback technology that enables training to be monitored by analysis of this voice dimension.

Finally, this general progress can only be achieved as the result of the type of concerted activity that was mentioned earlier in connection with the assessment of the GIRBAS scale. Multicentre, polylanguage enabling research can put the future efficient use of connected speech into the category of a reliable and simple calibrated everyday tool.

\section{Acknowledgements}

This work would not have been feasible without the critical input and the help of Evelyn Abberton and data and discussion, from and with: Elisabeth Fresnel, Julian McGlashan, Martin Ptok, Romain Pérouse, Bruno Coulombeau, the help of staff and students at RADA, LAMDA, University College London, and both hardware and software from Xinghui Hu (Laryngograph. com) and it would not have appeared without the incentive and forum for discussion provided by Philippe Dejonckere and other COST 2103 colleagues. Adrian Fourcin is a Director of Laryngograph Ltd. and a Trustee of Laryngograph Trust - a charity set up to support voice research.

Folia Phoniatr Logop 2009;61:126-136 


\section{References}

1 Fabre P: Un procédé électrique percutané d'inscription de l'accolement glottique au cours de la phonation. Bull Nat Méd 1957; 141:66-99.

-2 Fourcin A, Abberton E: Hearing and phonetic criteria in voice measurement: clinical applications. Logoped Phoniatr Vocol 2008;33: $35-48$.

3 Moore BCJ, Glasberg BR, Peters RW: Relative dominance of individual partials in determining the pitch of complex tones. J Acoust Soc Am 1985;75:550-561.

4 Nelson DA, Stanton ME, Freyman RL: A general equation describing frequency discrimination as function of frequency and sensation level. J Acoust Soc Am 1983;73: 2117-2123.

5 Vance TF: Variation in pitch discrimination within the tonal range, Iowa, 1914; in Boring EG (ed): Sensation and Perception in the History of Experimental Psychology. New York, Appleton-Century-Crofts, 1942, p 340.
6 Wier CC, Jesteadt W, Green DM: Frequency discrimination as a function of frequency and sensation level. J Acoust Soc Am 1977;61: 178-184.

7 Klatt DH: Discrimination of fundamental frequency contours in synthetic speech: implications for models of pitch perception. J Acoust Soc Am 1973;53:8-16.

8 't Hart J: Differential sensitivity to pitch distance, particularly in speech. J Acoust Soc Am 1981;65:811-821.

9 Buder EJ: Acoustic analysis of voice quality; in Kent RJ, Ball MJ (eds): Voice Quality Measurement. San Diego, Singular/Thompson Learning, 2000, pp 119-244.

10 Walzak P, McCabe P, Madill C, Sheard C: Acoustic changes in student actors' voices after 12 months of training. J Voice 2008;22: 257-384.
11 Ptok M, Iven C, Jessen M, Schemmle C: Objektivgemessene Stimmlippenschwingungsirregularitat vs. subjektiver Eindruck der Rauhigkeit. HNO 2006;54:132-136.

12 Hirano M: Clinical Examination of Voice. New York, Springer, 1981.

13 Dejonckere PH, Remacle M, Fresnel-Elbaz E, Woisard V, Crevier-Buchman L, Millet B: Differentiated perceptual evaluation of pathological voice quality: reliability and correlations with acoustic measurements. Rev Laryngol Otol Rhinol 1996;117:219224.

14 Abberton ER: Aspects of voice quality in women. Measurement of speech sound data and its practical application. Proc First Conf Speech Sci, Seoul, 2002, pp 128-131.

15 Sayles M, Winter I: Ambiguous pitch and the temporal representation of inharmonic iterated rippled noise in the ventral cochlear nucleus. J Neurosci 2008;28:11925-11938. 